\title{
ПЕРИОДИЧЕСКИЕ СОСТОЯНИЯ ВБЛИЗИ ПЛОСКОГО ДЕФЕКТА С НЕЛИНЕЙНЫМ ОТКЛИКОМ, РАЗДЕЛЯЮЩЕГО НЕЛИНЕЙНЫЙ САМОФОКУСИРУЮЩИЙ И ЛИНЕЙНЫЙ КРИСТАЛЛЫ
}

\author{
(C) 2018 C. Е. Савотченко \\ Белгородский государственный технологический университет им. В. Г. Шухова, \\ ул. Костюкова, 46, 308012 Белгород, Россия \\ e-mail: savotchenkose@mail.ru
}

Поступила в редакцию: 26.03.2018

\begin{abstract}
Аннотация. Предложена модель, описывающая особенности распространения возбуждений вблизи границы раздела линейной среды и нелинейной среды с положительной нелинейностью, которая представляет собой плоский дефект с внутренними нелинейными свойствами. В основе модели лежит нелинейное уравнение Шредингера с нелинейным самосогласованным потенциалом. Показано, что в рассматриваемой системе существуют нелинейные пространственно-неоднородные состояния нескольких типов, определяемые различными периодическими решениями нелинейного уравнения Шредингера. Получены и проанализированы дисперсионные соотношения, определяющие энергию таких стационарных состояний. Установлено, что в спектре существуют резонансные состояния, обусловленные исключительно нелинейными свойствами дефекта. Получены добавки к спектральной плотности состояний и указаны ее характерные особенности.
\end{abstract}

Ключевые слова: нелинейное уравнение Шредингера, граница раздела сред, плоский дефект, периодические решения, нелинейные волны, плотность состояний, локализованные состояния.

DOI: https://doi.org/10.17308/kcmf.2018.20/517

\section{ВВЕДЕНИЕ}

Построение моделей границ раздела сред с различными физическими свойствами имеет важное значения как с точки зрения развития фундаментальных исследований, так и с точки зрения разработки технических приложений в микроэлектронике и оптоэлектронике. Использование свойств границ раздела сред является актуальным в связи с широким применением контролируемых барьеров. Здесь особую важность приобретают исследования закономерностей локализации возбуждений вблизи границ раздела сред.

Если рассматриваются возбуждения электромагнитной природы или оптического диапазона в различных многослойных гетероструктурах, состоящих из чередующихся диэлектрических и металлических слоев или слоев из магнетиков различных типов, используются модели линейных и нелинейных сред. На границах между слоями возбуждения терпят различные преобразования, которые обусловлены характером их взаимодействия с границей как с плоским дефектом кристаллической структуры.

Теоретические исследования нелинейных поверхностных волн электромагнитной природы проводятся достаточно давно [1] и остаются актуальными до сих пор в связи с их широким применением в оптических системах хранения данных [2]. Вблизи границ раздела нелинейной и линейной сред теоретически была показана возможность существования нелинейных поверхностных электромагнитных волн [3].

Широкое применение в такого рода исследованиях находит нелинейное уравнение Шредингера (НУШ). НУШ использовалось для описания звуковых нелинейных поверхностных волн и солитонов в кристаллах [4], распространения малоамплитудных спиновых волн [5], распространения деформационных импульсов в молекулярных цепочках [6]. В связи с этим для описания новых эффектов, обусловленных взаимодействием возбуждений различной физической природы с плоскими дефекта- 


\section{С. Е. САВОТЧЕНКО}

ми кристаллической структуры, имеет смысл далее рассматривать математическую модель, основанную на НУШ [7].

При построении моделей плоских границ раздела сред применяется короткодействующий потенциал, в одномерном случае содержащий дельта-функцию Дирака. На основе системы НУШ с потенциалом такого вида в [8] рассмотрено взаимодействие вблизи дефекта связанных солитонных состояний, относящихся к различным состояниям двухуровневой системы в нелинейных средах, а в [9] - для границы раздела линейных и нелинейных сред. Проводился также учет влияния пространственной дисперсии на локализацию возбуждений [10]. Новые особенности локализации состояний вблизи границ раздела нелинейных сред и линейной и нелинейной сред с учетом внутренней структуры дефекта рассмотрены в [11-13].

В данной работе предлагается обобщение модели, описывающей процессы локализации и трансформации возбуждений на границе раздела линейной и нелинейной сред [14], на случай когда она обладает собственными нелинейными свойствами, способными оказывать влияние на профиль поля и спектр возбуждений. Нелинейные свойства дефекта учитываются в модели посредством потенциала, использованного в [15-18] для описания эффектов локализации колебаний. Целью данной работы является нахождение новых типов пространственнонеоднородных периодических состояний вблизи плоского дефекта с нелинейным откликом, разделяющим линейный кристалл и нелинейную среду с взаимным притяжением, их энергии и спектральные плотности.

\section{1. УРАВНЕНИЯ МОДЕЛИ}

Будем рассматривать процессы взаимодействия возбуждений на границе раздела линейной и нелинейной сред на основе уравнения Шредингера:

$$
\begin{gathered}
i \frac{\partial \psi}{\partial t}=-\frac{1}{2 m} \frac{\partial^{2} \psi}{\partial x^{2}}+\Omega(x) \psi- \\
-\gamma \theta(x)|\psi|^{2} \psi+\psi\left\{U_{0}+W_{0}|\psi|^{2}\right\} \delta(x),
\end{gathered}
$$

где использована тета-функция Хевисайда:

$$
\theta(x)=\left\{\begin{array}{l}
0, x<0 \\
1, x>0
\end{array},\right.
$$

$m$ - эффективная масса возбуждений,

$$
\Omega(x)=\left\{\begin{array}{l}
\Omega_{1}, x<0 \\
\Omega_{2}, x>0
\end{array},\right.
$$

$\Omega_{1,2}$ - значения уровней дна энергетический зоны (границы сплошного спектра линейных волн), $\gamma$ - параметр нелинейности среды, расположенной справа от дефекта, $\delta(x)-\delta$-функция Дирака, $U_{0}-$ интенсивность взаимодействия плоского дефекта, проходящего через начало координат, с возбуждением (при $U_{0}>0$ возбуждение отталкивается от дефекта, при $U_{0}<0$ - притягивается), $W_{0}-$ интенсивность нелинейного отклика дефекта, положительное значение которого соответствует отталкиванию внутри тонкого дефектного слоя, а отрицательное - притяжению. Если рассматриваются нелинейные оптические возбуждения в волноводах, то принято называть нелинейные керровские среды с притяжением оптическими средами с самофокусировкой, а среды с отталкиванием - средами с дефокусировкой.

Если в уравнение (1) подставить волновую функцию в виде $\psi(x, t)=\psi(x) \exp (-i E t)$, где $E$ - энергия стационарных состояний, то можно рассматривать два стационарных уравнения на полуосях без потенциала. Слева от границы раздела получается стационарное линейное УШ, а справа - стационарное НУШ:

$$
\begin{gathered}
\frac{1}{2 m} \psi^{\prime \prime}(x)+\left(E-\Omega_{1}\right) \psi(x)=0, x<0, \\
\frac{1}{2 m} \psi^{\prime \prime}(x)+\left(E-\Omega_{2}\right) \psi(x)+\gamma|\psi(x)|^{2} \psi(x)=0, \\
x>0,
\end{gathered}
$$

с граничными условиями:

$$
\begin{aligned}
\psi(-0) & =\psi(+0)=\psi_{0} \\
\psi^{\prime}(+0)-\psi^{\prime}(-0) & =2 m \psi_{0}\left\{U_{0}+W_{0}\left|\psi_{0}\right|^{2}\right\} .
\end{aligned}
$$

В НУШ (1) использован нелинейный потенциал $U(x)=\left\{U_{0}+W_{0}\left|\psi_{0}\right|^{2}\right\} \delta(x)$, описывающий нелинейные свойства внутри бесконечно тонкого плоского дефектного слоя, разделяющего кристаллы [15]. Из него, как следствие, получается нелинейное граничное условие (5). При $U_{0}=0$ оно применялось в $[16,17]$ для описания локализации состояния и анализа их устойчивости в ангармонической среде с нелинейным дефектом.

В отсутствие границы раздела $(U(x) \equiv 0)$ в нелинейном кристалле с положительной нелинейностью $(\gamma>0)$ и одинаковых параметрах сред $\Omega_{j}=\Omega, j=1,2$, существуют два типа стационарных состояний при $E<\Omega$, описываемых двумя типами периодических решений НУШ, представляющих собой нелинейные (кноидальные) волны стационарного профиля [6]:

1) $\psi(x)=k q_{c}(m \gamma)^{-1 / 2} \operatorname{cn}\left(q_{c}\left(x-x_{0}\right), k\right)$, с энергией: $E=\Omega-q_{c}^{2}\left(2 k^{2}-1\right) / 2 m$, где $k-$ модуль эллип- 
тической функции $\mathrm{cn}\left(1 / 2<k^{2}<1\right), k, q_{c}$ и $x_{0}$ являются свободными параметрами;

2) $\psi(x)=q_{d}(m \gamma)^{-1 / 2} \operatorname{dn}\left(q_{d}\left(x-x_{0}\right), k\right)$, с энергией: $E=\Omega-q_{d}^{2}\left(2-k^{2}\right) / 2 m$, где $k-$ модуль эллиптической функции $\operatorname{dn}\left(0<k^{2}<1\right), k, q_{d}$ и $x_{0}$ являются свободными параметрами.

При $k=1$ оба таких периодических решения переходят в соответствии со свойствами эллиптических функций в одно решение, представляющее собой солитон: $\psi(x)=q(m \gamma)^{-1 / 2} / \operatorname{ch} q\left(x-x_{0}\right)$, с максимумом в точке $x=x_{0}$, с энергией $E=\Omega-q^{2} / 2 m$ , волновое число $q$ и $x_{0}$ являются свободными параметрами.

\section{2. ПРОСТРАНСТВЕННО-НЕОДНОРОДНЫЕ ПЕРИОДИЧЕСКИЕ СОСТОЯНИЯ}

При наличии плоского дефекта, разделяющего линейный кристалл и нелинейный кристалл с взаимным притяжением, соответствующей положительной нелинейности НУШ $\gamma>0$, возможно существование двух типов стационарных состояний с энергией внутри сплошного спектра в диапазоне $\Omega_{1}<E<\Omega_{2}$. Следует отметить, что пространственно-неоднородные периодические состояния существуют в случае, когда $\Omega_{1}<\Omega_{2}$. Данное требование означает, что уровень края сплошного спектра в линейном кристалле должен быть ниже, чем в нелинейном.

\section{1. Периодические состояния 1-го типа}

Стационарное состояние первого типа описывается решением уравнений (2) и (3):

$$
\psi(x)=\left\{\begin{array}{l}
B_{c} \cos (p x+\varphi), x<0 \\
A_{c} \operatorname{cn}\left(q_{c}\left(x-x_{0}\right), k\right), x>0
\end{array}\right.
$$

Подстановка решения (6) в области $x<0$ в УШ (2) позволяет получить волновое число:

$$
p^{2}=2 m\left(E-\Omega_{1}\right) .
$$

Подстановка решения (6) в области $x>0$ в НУШ (3) позволяет получить волновое число и амплитуду нелинейной волны:

$$
\begin{gathered}
q_{c}^{2}=2 m\left(\Omega_{2}-E\right) /\left(2 k^{2}-1\right), \\
A_{c}^{2}=k^{2} q_{c}^{2} /(m \gamma) .
\end{gathered}
$$

Волновые числа (7) и (8) связаны соотношением:

$$
p^{2}=2 m\left(\Omega_{2}-\Omega_{1}\right)-q_{c}^{2}\left(2 k^{2}-1\right) .
$$

Подстановка решения (6) в условие непрерывности (4) позволяет получить амплитуду линейной волны:

$$
B_{c}=k q_{c}(m \gamma)^{-1 / 2} \operatorname{cn}\left(q_{c} x_{0}, k\right) / \cos \varphi .
$$

Подстановка решения (6) в нелинейное граничное условие (5) приводит к дисперсионному соотношению:

$$
p \operatorname{tg} \varphi=\Delta_{c},
$$

где

$$
\begin{gathered}
\Delta_{c}=2\left\{m U_{0}+W_{0} k^{2} q_{c}^{2} \mathrm{cn}^{2}\left(q_{c} x_{0}, k\right) / \gamma\right\}- \\
-q_{c} \operatorname{sn}\left(q_{c} x_{0}, k\right) / \operatorname{sn}\left(q_{c} x_{0}+K(k), k\right),
\end{gathered}
$$

$K(k)$ - полный эллиптический интеграл первого рода.

Соотношение (12) далее будем называть дисперсионным, поскольку из него определяется энергия стационарного состояния (6) как функция параметров кристаллов и дефекта: $E=E\left(m, U_{0}, W_{0}, \gamma\right.$, $\left.\Omega_{1,2}, x_{0}, k, \varphi\right)$. Величины $k, \varphi$ и $x_{0}$ являются свободными параметрами.

Воспользовавшись дисперсионным соотношением (12), можно исключить фазу ф из (11) и получить амплитуду линейной волны в виде:

$$
B_{c}=k q_{c}\left\{\left(p^{2}+\Delta_{c}^{2}\right) / m \gamma\right\}^{1 / 2} \operatorname{cn}\left(q_{c} x_{0}, k\right) / p .
$$

Можно отметить, что существует выделенный уровень энергии при $\varphi=0$. Такой энергией обладает волна, амплитуда колебаний $B_{c}$ которой совпадает с амплитудой колебаний плоского дефекта $\psi_{0}$. В таком смысле его можно назвать резонансным. Уровень энергии такого резонансного состояния определяется из дисперсионного соотношения (12), которое при $\varphi=0$ принимает вид: $\Delta_{c}=0$.

\section{2. Периодические состояния 2-го типа}

Стационарное состояние второго типа описывается решением уравнений (2) и (3):

$$
\psi(x)=\left\{\begin{array}{l}
B_{d} \cos (p x+\varphi), x<0 \\
A_{d} \operatorname{dn}\left(q_{d}\left(x-x_{0}\right), k\right), x>0
\end{array} .\right.
$$

Волновое число $p$ линейной волны определяется выражением (7). Подстановка решения (14) в области $x>0$ в НУШ (3) позволяет получить волновое число и амплитуду нелинейной волны:

$$
\begin{gathered}
q_{d}^{2}=2 m\left(\Omega_{2}-E\right) /\left(2-k^{2}\right), \\
A_{d}^{2}=q_{d}^{2} /(m \gamma) .
\end{gathered}
$$

Волновые числа (7) и (15) связаны соотношением:

$$
p^{2}=2 m\left(\Omega_{2}-\Omega_{1}\right)-q_{d}^{2}\left(2-k^{2}\right) .
$$

Подстановка решения (14) в условие непрерывности (4) позволяет получить амплитуду линейной волны:

$$
B_{d}=q_{d}(m \gamma)^{-1 / 2} \operatorname{dn}\left(q_{d} x_{0}, k\right) .
$$

Подстановка решения (6) в нелинейное граничное условие (5) приводит к дисперсионному соотношению: 


\section{С. Е. САВОТЧЕНКО}

$$
p \operatorname{tg} \varphi=\Delta_{d}
$$

где

$$
\begin{gathered}
\Delta_{d}=2\left\{m U_{0}+W_{0} q_{d}^{2} \operatorname{dn}^{2}\left(q_{d} x_{0}, k\right) / \gamma\right\}- \\
-k^{2} q_{d} \operatorname{sn}\left(q_{d} x_{0}, k\right) \operatorname{sn}\left(q_{d} x_{0}+K(k), k\right) .
\end{gathered}
$$

Соотношение (19) также будем называть дисперсионным, поскольку из него определяется энергия стационарного состояния (14) как функция параметров кристаллов и дефекта.

Уровень энергии резонансного состояния, при котором амплитуда линейной волны $B_{d}$ совпадает с амплитудой колебаний плоского дефекта $\psi_{0}$, определяется из дисперсионного соотношения (19) при $\varphi=0$, которое принимает вид: $\Delta_{d}=0$.

При $W_{0}=0$ дисперсионные соотношения (12) и (19) переходят в выражения, полученные в [14] для описания энергии трансформации нелинейных волн соответственно первого и второго типов при переходе в линейный кристалл через границу с простым линейным взаимодействием с возбуждением. Если дополнительно положить $k=1$, то указанные соотношения переходят в полученные в работе [19] дисперсионные соотношения для описания процессов локализации. Случай локальных состояний вблизи нелинейного дефекта, разделяющего две нелинейных среды (когда $U_{0}=0, W_{0} \neq 0$ ) был рассмотрен в [18].

В явном виде можно получить энергию стационарных состояний из дисперсионных соотношений в различных частных случаях.

\section{3. ЭНЕРГИИ ПРОСТРАНСТВЕННО- НЕОДНОРОДНЫХ ПЕРИОДИЧЕСКИХ СОСТОЯНИЙ}

\section{1. Энергия периодических состояний 1-го типа}

Дисперсионное уравнение имеет точное решение в случае, когда $x_{0}=0$. Тогда для произвольных значений $\varphi \neq 0$ из (12) получается выражение для энергии в виде:

$$
E=\Omega_{2}-\Omega_{c 0}\left\{1 \pm\left[1-\Omega_{c a} / \Omega_{c 0}\right]^{1 / 2}\right\},
$$

где

$$
\begin{gathered}
\Omega_{c 0}=-\gamma\left(2 k^{2}-1\right) Q_{c} / 16 m W_{0}^{2} k^{4}, \\
\Omega_{c a}=2 \gamma\left(2 k^{2}-1\right) Q_{0} / Q_{c}, \\
Q_{c}=\gamma\left(2 k^{2}-1\right) \operatorname{tg}^{2} \varphi+8 m U_{0} W_{0} k^{2}, \\
Q_{0}=\left(\Omega_{2}-\Omega_{1}\right) \operatorname{tg}^{2} \varphi-2 m U_{0}^{2} .
\end{gathered}
$$

Уровень энергии резонансного состояния определяется из (20) при $\varphi=0$ :

$$
E=\Omega_{2}+\frac{\gamma U_{0}\left(2 k^{2}-1\right)}{2 W_{0} k^{2}} .
$$

Из (21) видно, что такое резонансное состояние возможно только при отличных от нуля и противоположных по знаку параметрах дефекта $U_{0}$ и $W_{0}$.

Для случая $x_{0} \neq 0$ и $\varphi=0$ из (12) в явном аналитическом виде можно найти энергию в «длинноволновом» приближении при $q_{c} x_{0} \ll 1$. Условие «длинноволнового» приближения означает близость энергии возбуждения к краю спектра, когда выполняется требование $\left|\Omega_{2}-E\right|<<\left(2 k^{2}-1\right) / 2 m x_{0}^{2}$ . В таком пределе в основном приближении из (12) получается:

$$
E=\Omega_{2}-\frac{\gamma U_{0}\left(2 k^{2}-1\right)}{\gamma x_{0}-2 W_{0} k^{2}} .
$$

Из (22) следует, что для существования резонансных состояний такого вида должна выполняться одна из пар требований: 1) $U_{0}>0$ и $W_{0}<\gamma x_{0} / 2 k^{2}$ или 2) $U_{0}<0$ и $W_{0}>\gamma x_{0} / 2 k^{2}$. Видно, что в отличие от резонансного состояния, энергия которого определяется выражением (21), резонансные состояния с энергией (22) могут существовать при одинаковых знаках дефекта, если знак параметра $x_{0}$ противоположен знаку параметра дефекта $U_{0}$.

Если $x_{0} \neq 0$ и $\varphi \neq 0$, то из (12) в «длинноволновом» приближении при $q_{c} x_{0}<<1$ получается выражение для энергии в виде:

$$
E=\Omega_{2}-\Omega_{c 0}^{x}\left\{1 \pm\left[1-\Omega_{c a}^{x} / \Omega_{c 0}^{x}\right]^{1 / 2}\right\},
$$

где

$$
\begin{gathered}
\Omega_{c 0}^{x}=-\gamma\left(2 k^{2}-1\right) Q_{c}^{x} / 4 m\left(\gamma x_{0}-2 W_{0} k^{2}\right)^{2}, \\
\Omega_{c a}^{x}=2 \gamma\left(2 k^{2}-1\right) Q_{0} / Q_{c}^{x}, \\
Q_{c}^{x}=\gamma\left(2 k^{2}-1\right) \operatorname{tg}^{2} \varphi+4 m U_{0}\left(\gamma x_{0}-2 W_{0} k^{2}\right) .
\end{gathered}
$$

Выражение (23) переходит: 1) при $x_{0}=0$ и $\varphi \neq 0$ в $(20) ; 2)$ при $x_{0} \neq 0$ и $\varphi=0$ в $\left.(22) ; 3\right)$ при $x_{0}=0$ и $\varphi=0$ B (21).

\section{2. Энергия периодических состояний 2-го типа}

Дисперсионное уравнение имеет точное решение в случае, когда $x_{0}=0$. Тогда для произвольных значений $\varphi \neq 0$ из (19) получается выражение для энергии в виде:

$$
\begin{gathered}
E=\Omega_{2}-\Omega_{d 0}\left\{1 \pm\left[1-\Omega_{d a} / \Omega_{d 0}\right]^{1 / 2}\right\}, \\
\Omega_{d 0}=-\gamma\left(2-k^{2}\right) Q_{d} / 16 m W_{0}^{2}, \\
\Omega_{d a}=2 \gamma\left(2-k^{2}\right) Q_{0} / Q_{d}, \\
Q_{d}=\gamma\left(2-k^{2}\right) \operatorname{tg}^{2} \varphi+8 m U_{0} W_{0} .
\end{gathered}
$$

где

Уровень энергии резонансного состояния определяется из (24) при $\varphi=0$ :

$$
E=\Omega_{2}+\frac{\gamma U_{0}\left(2-k^{2}\right)}{2 W_{0} k^{2}} .
$$


Из (25) видно, что такое резонансное состояние возможно только при противоположных знаках параметров дефекта $U_{0}$ и $W_{0}$, и когда оба они отличны от нуля.

Для случая $x_{0} \neq 0$ и $\varphi=0$ из (19) в явном аналитическом виде энергию можно найти в «длинноволновом» приближении при $q_{d} x_{0} \ll 1$. Условие «длинноволнового» приближения означает близость энергии возбуждения к краю спектра, когда выполняется требование $\left|\Omega_{2}-E\right|<<\left(2-k^{2}\right) / 2 m x_{0}^{2}$ . В таком пределе в основном приближении из (19) получается:

$$
E=\Omega_{2}-\frac{\gamma U_{0}\left(2-k^{2}\right)}{\gamma x_{0} k^{2}-2 W_{0}}
$$

Из (26) следует, что для существования резонансных состояний такого вида должна выполняться одна из пар требований: 1) $U_{0}>0$ и $W_{0}<\gamma x_{0} k^{2} / 2$ или 2) $U_{0}<0$ и $W_{0}>\gamma x_{0} k^{2} / 2$. Видно, что в отличие от резонансного состояния, энергия которого определяется выражением (25), резонансные состояния с энергией (26) могут существовать при одинаковых знаках дефекта, если знак параметра $x_{0}$ противоположен знаку параметра дефекта $U_{0}$.

Если $x_{0} \neq 0$ и $\varphi \neq 0$, то из (19) в «длинноволновом» приближении при $q_{d} x_{0} \ll 1$ получается выражение для энергии в виде:

$$
E=\Omega_{2}-\Omega_{d 0}^{x}\left\{1 \pm\left[1-\Omega_{d a}^{x} / \Omega_{d 0}^{x}\right]^{1 / 2}\right\},
$$

где

$$
\begin{gathered}
\Omega_{d 0}^{x}=-\gamma\left(2-k^{2}\right) Q_{d}^{x} / 4 m\left(2 W_{0}-\gamma x_{0} k^{2}\right)^{2}, \\
\Omega_{d a}^{x}=2 \gamma\left(2-k^{2}\right) Q_{0} / Q_{d}^{x}, \\
Q_{d}^{x}=\gamma\left(2-k^{2}\right) \operatorname{tg}^{2} \phi+4 m U_{0}\left(2 W_{0}-\gamma x_{0} k^{2}\right) .
\end{gathered}
$$

Выражение (27) переходит: 1) при $x_{0}=0$ и $\varphi \neq 0$ в (24);2) при $x_{0} \neq 0$ и $\varphi=0$ в $\left.(26) ; 3\right)$ при $x_{0}=0$ и $\varphi=0$ в (25).

Следует отметить, что резонансные состояния рассмотренных типов с энергиями (21) и (25) могут возникать только вблизи плоского дефекта с нелинейным откликом, поскольку при $W_{0}=0$ они не существуют.

\section{4. ПЛОТНОСТЬ СОСТОЯнИЙ}

Зависимость фазы от энергии $\varphi=\varphi(E)$ позволяет получить добавку к плотности состояний, возникающую за счет дефекта [19-23]:

$$
\delta g(E)=\frac{1}{\pi} \frac{\partial \varphi}{\partial E} .
$$

Для упрощения вычислений далее будем рассматривать только такие стационарные состояния, для которых параметр $x_{0}=0$.

\section{1. Плотность периодических состояний 1-го типа}

Из дисперсионного соотношения (12) выражается

$$
\varphi=\operatorname{arctg}\left(\Delta_{c} / p\right) .
$$

Подставив (29) в (28) при $x_{0}=0$,можно получить плотность состояний в виде:

$$
\delta g_{c}(E)=\delta g_{1 c}(E)+\delta g_{2 c}(E),
$$

где

$$
\begin{gathered}
\delta g_{1 c}(E)=-\frac{m \Delta_{c 0}}{\pi p\left(p^{2}+\Delta_{c 0}^{2}\right)}, \\
\delta g_{2 c}(E)=-\frac{4 m W_{0} k^{2} p}{\pi \gamma q_{c}\left(2 k^{2}-1\right)\left(p^{2}+\Delta_{c 0}^{2}\right)}, \\
\Delta_{c 0}=2\left\{m U_{0}+W_{0} k^{2} q_{c}^{2} / \gamma\right\} .
\end{gathered}
$$

В эти выражения подставляются зависимости (7) и (8).

Можно отметить следующие особенности плотности состояний (30).

1) Если выполняется требование $q_{c}^{2}<-\gamma m U_{0} / W_{0} k^{2}$, которое реализуется только при противоположных знаках параметров дефекта $U_{0}$ и $W_{0}$, то $\delta g_{1 c}(E)>0$.

2) Если дефект является простым без нелинейного отклика, то есть когда $W_{0}=0$, то второе слагаемое в (30) обращается в ноль: $\delta g_{2 c}(E)=0$. Добавка $\delta g_{2 A}(E)$ характеризует нелинейные свойства дефекта.

3) Плотности состояний (30) имеет характерные для одномерных моделей корневые особенности на краях сплошного спектра.

4) Полюсы плотности состояний (30) определяются из требования $\Delta_{c}^{2}=-p^{2}$, реализация которого возможна только при комплексном (а точнее - мнимом для монотонного затухания поля при удалении от плоскости дефекта) волновом числе, то есть когда $p=i \kappa$, что приводит к дисперсионному соотношению для локализованных состояний. Для локализованных состояний величина пространственного затухания определяется выражением: $\kappa=-\Delta_{c 0}$.

\section{2. Плотность периодических состояний 2-го типа}

Из дисперсионного соотношения (19) выражается

$$
\varphi=\operatorname{arctg}\left(\Delta_{d} / p\right) .
$$

Подставив (31) в (28) при $x_{0}=0$, можно получить плотность состояний в виде:

$$
\delta g_{d}(E)=\delta g_{1 d}(E)+\delta g_{2 d}(E),
$$




\section{С. Е. САВОТЧЕНКО}

где

$$
\begin{gathered}
\delta g_{1 d}(E)=-\frac{m \Delta_{d 0}}{\pi p\left(p^{2}+\Delta_{d 0}^{2}\right)}, \\
\delta g_{2 d}(E)=-\frac{4 m W_{0} p}{\pi \gamma q_{d}\left(2-k^{2}\right)\left(p^{2}+\Delta_{d 0}^{2}\right)}, \\
\Delta_{d 0}=2\left\{m U_{0}+W_{0} q_{d}^{2} / \gamma\right\} .
\end{gathered}
$$

В эти выражения подставляются зависимости (7) и (15).

Можно отметить следующие особенности плотности состояний (32).

1) Если выполняется требование $q_{d}^{2}<-\gamma m U_{0} / W_{0}$ , которое реализуется только при противоположных знаках параметров дефекта $U_{0}$ и $W_{0}$, то $\delta g_{1 d}(E)>0$.

2) Плотности состояний (32) имеет характерные для одномерных моделей корневые особенности на краях сплошного спектра.

3) Если дефект является простым без нелинейного отклика, то есть когда $W_{0}=0$, то второе слагаемое в (32) обращается в ноль. Добавка $\delta g_{2 d}(E)$ характеризует нелинейные свойства дефекта.

4) Полюсы плотности состояний (32) определяются из требования $\Delta_{d}^{2}=-p^{2}$, реализация которого возможна только при $p= \pm i \kappa$, что приводит к дисперсионному соотношению для локализованных состояний: $\kappa=-\Delta_{d 0}$, определяющего энергию локализации стационарного состояния.

Соответствующие дисперсионные соотношения для локализованных в нелинейных средах состояний вблизи нелинейного дефекта (когда $U_{0}=0$, $\left.W_{0} \neq 0\right)$ были получены в [18].

\section{ЗАКЛЮЧЕНИЕ}

В работе получены связанные периодические решения линейного и нелинейного УШ на полуосях, описывающие взаимодействие возбуждений на границах раздела линейной и нелинейной сред, которая обладает внутренними нелинейными свойствами. Проанализирован случай нелинейной среды с взаимным притяжением, которой соответствует положительное значение параметра нелинейности НУШ. Для описания нелинейного отклика дефекта предложено использовать нелинейный самосогласованный потенциал в короткодействующем приближении. Данный потенциал характеризуется двумя параметрами: интенсивностью взаимодействия границы раздела с возбуждением в линейном приближении и параметром нелинейности, знак которого определяет притяжение и отталкивание в самой границе.

Показано, что в среде с положительной нелинейностью существуют два типа пространственно- неоднородных периодических состояний, описываемых отличающимися в полупространстве с нелинейной средой решениями НУШ, которые выражаются через различные эллиптические функции. Данные состояния описывают трансформацию нелинейных волн соответственно первого и второго типов при переходе в полупространство с линейной средой через границу с нелинейным взаимодействием с возбуждениями. Для каждого типа состояний получены дисперсионные соотношения, определяющие их спектр в виде зависимости энергии от характеристик сред и дефекта. Установлено, что существует выделенный уровень энергии (при $\varphi=0)$, называемый резонансным, при котором амплитуда линейной волны совпадает с амплитудой колебаний плоского дефекта.

Получены точные решения дисперсионных уравнений, определяющие энергии колебательных состояний частного вида (при $\left.x_{0}=0\right)$. Найдены выражения для энергии длинноволновых колебательных состояний общего вида (при $x_{0} \neq 0$ ). Указаны условия их существования. Следует отметить, что резонансные состояния рассмотренных типов является новыми, характерными только для плоского дефекта с нелинейным откликом.

Рассчитаны добавки к плотности состояний, возникающие за счет дефекта, и проанализированы их свойства. Отмечено, что полюсы плотности состояний определяют спектр локализованных вблизи границы раздела сред колебательных состояний. Добавки к плотности состояний представлены в виде двух слагаемых, второе из которых характеризует исключительно нелинейный отклик дефекта.

Таким образом, новизна работы заключается в том, что учет нелинейного отклика в модели плоского дефекта позволяет описать новые типы резонансных колебательных состоянии и привносит дополнительные особенности в спектральную плотность колебательных состояний.

\section{СПИСОК ЛИТЕРАТУРЫ}

1. Михалаке Д., Назмитдинов Р. Г., Федянин В. К. // Физика элементарных частиц и атомного ядра, 1989, т. 20, № 1, с. 198-253.

2. Паняев И. С., Санников Д. Г. // Компьютерная оптика, 2017, т. 41, с. 183-191.

3. Ахмедиев Н. Н., Корнеев В. И., Кузьменко Ю. В. // ЖЭТФ, 1985, т. 88, № 1, с. 107-115.

4. Горенцвейг В. И., Кившарь Ю. С., Косевич А. М., Сыркин Е. С. // ФНТ, 1990, т. 16, с. 1472-1482.

5. Герасимчук И. В., ГоробецЮ.И., Герасимчук В. С. // Journal of Nano- and Electronic Physics, 2016, т. 2, c. 02020-1-7. DOI: 10.21272/jnep.8(2).02020 
6. Давыдов А. С. Солитоны в молекулярных системах. Киев: Наукова думка, 1984, 288 с.

7. Герасимчук И. В., Ковалев А. С. // Физика низких meмnepamyp, 2000, т. 26, № 8, с. 799-809.

8. Савотченко С. Е. // Конденсированные среды и межфазные гранииьы, 2017, т. 19, № 2, с. 291-259.

9. Савотченко С. Е. // ЖТФ, 2017, т. 62, № 12 , c. 1776-1781.DOI: https://doi:10.21883/JTF.2017.12. 45197.2282

10. Савотченко С. Е. // Известия высших учебных заведений. Физика, 2004, т. 47, № 5, с. 79-84.

11. Савотченко С. Е. // Вестник Воронежского государственного университета. Серия: Физика. Математика, 2016, № 4, с. 51-59.

12. Савотченко С. Е. // Научные ведомости БелГУ. Сер.: Математика. Физика, 2017, № 20(269), c. $79-85$.

13. Савотченко С. Е. // ЖЭТФ, 2018, т. 153, № 2, c. 339-348. DOI: https://doi:10.7868/S0044451018020153

14. Савотченко С. Е. // Вестник Воронежского государственного университета. Серия: Физика. Математика, 2018, № 1, с. 42-51.
15. Gerasimchuk I. V. // Journal of Nano- and Electronic Physics, 2012, vol. 4, № 4, p. 04024-1-4.

16. Gerasimchuk I. V., Gorbach P. K., Dovhopolyi P. P. // Ukr. J. Phys., 2012, vol. 57, № 6, pp. 678-683.

17. Герасимчук И. В. // ЖЭТФ, 2015, т. 121, № 4, c. 596-605. DOI: https://doi:10.7868/ S0044451015100053

18. Savotchenko S. E. // Modern Physics Letters B, 2018, vol. 32, № 10, pp. 1850120-12. DOI: https://doi. org/10.1142/S0217984918501208

19. Савотченко С. Е. // Конденсированные среды и межфазные границьы, 2017, т. 19, № 4, с. 567-572.

20. Косевич А. М. // ЖЭТФ, 1999, т. 115, № 1, c. 306-317.

21. Косевич А. М., Мацокин Д. В., Савотченко С. Е. // Письма в ЖЭТФ, 2001, т. 73, № 11-12, с. 680-683.

22. Савотченко С. Е. // Известия высших учебных заведений. Физика, 2001, т. 44, № 4, с. 67-73. DOI: https:// doi:10.1023/A:1011952514072

23. Савотченко С. Е. // Известия высших учебных заведений. Физика, 2002, т. 45, № 12, с. 1148-1158. DOI: https://doi:10.1023/A:1023858101297

\title{
PERIODIC STATES NEAR THE PLANE DEFECT WITH NON-LINEAR RESPONSE SEPARATING NON-LINEAR SELF-FOCUSING AND LINEAR CRYSTALS
}

\author{
(C) 2018 S. E. Savotchenko
}

Belgorod State Technological University named after V.G. Shukhov, 46 Kostyukova str., 308012 Belgorod, Russia e-mail:savotchenkose@mail.ru

Received 26.03.2018

\begin{abstract}
The paper presents a model describing the peculiarities of localised excitation on the boundary between linear and nonlinear self-focusing media. The medium boundary is a plane defect with internal nonlinear properties. The boundary of nonlinear media, characterised by various parameters of anharmonicity of the interatomic interaction, creates a disturbance of the medium characteristics, which is located at distances much smaller than the width of the localization of propagating waves. The model is based on the nonlinear Schrödinger equation with a nonlinear self-consistent potential. The problem is reduced to the solution of the linear and nonlinear Schrödinger equations on half-spaces coupled by the boundary conditions. The nonlinearity in the Schrödinger equation is assumed to be of the Kerr type with a positive parameter. Explicit solutions of nonlinear Schrödinger equations satisfying the boundary conditions were found for positive and negative nonlinearity parameters. It is shown that the existence of nonlinear spatially inhomogeneous states of several types determined by various periodic solutions of the nonlinear Schrödinger equation is possible in the system under consideration. The dispersion relations determining the energy of such stationary states were obtained and analysed. The energy dependences on the system parameters for stationary states in various limiting cases were obtained in an explicit form. It was established that resonance states exist in the spectrum, determined exclusively by the nonlinear properties of the defect. The additions to the spectral density of states were obtained, and its characteristic features were determined.
\end{abstract}

Keywords: nonlinear Schrödinger equation, boundary media, plane defect, periodic solutions, nonlinear waves, density of states, localized states.

DOI: https://doi.org/10.17308/kcmf.2018.20/517 


\section{С. Е. САВОТЧЕНКО}

\section{REFERENCES}

1. Mihalake D., Nazmitdinov R. G., Fedyanin V. K. Physics of Elementary Particles and the Atomic Nucleus, 1989, vol. 20, no. 1, pp. 198-253.(in Russ.)

2. Panyayev I. S., Sannikov D. G. Computer Optics, 2017, vol. 41, pp. 183-191. (in Russ.)

3. Ahmediev N. N., Korneev V. I., Kuzmenko U. V. Sov. Phys. JETP, 1985, vol. 61, no. 1, pp. 62-67. Available at: http://www.jetp.ac.ru/cgi-bin/dn/e_061_01_0062.pdf

4. Gorentsveig V. I., Kivshar Yu. S., Kosevich A. M., Syrkin E. S. Sov. Low Temp. Phys., 1990, vol. 16, p. 14721482. (in Russ.)

5. Gerasimchuk I. V., Gorobets Yu. I., Gerasimchuk V. S. Journal of Nano- and Electronic Physics, 2016, vol. 2, pp. 02020-1-7. DOI: 10.21272/jnep.8(2).02020

6. Davydov A. S. Solitons in Molecular Systems. Kiev, Naukova Dumka Publ., 1984, 288 p. (in Russ.)

7. Gerasimchuk I. V., Kovalev A. S. Low Temp. Phys., 2000, vol. 26, no. 8, pp. 799-809. (in Russ.)

8. Savotchenko S. E. Rus. Condensed Matter and Interphases, 2017, vol. 19, no. 2, pp. 291-259. Available at: http://www.kcmf.vsu.ru/article.php?1=ru\&aid=823 (in Russ.)

9. Savotchenko S. E. Sov. Technical Physics. 2017, vol. 62, no. 12, pp. 1776-1781. DOI: https://doi:10.21883/ JTF.2017.12.45197.2282

10. Savotchenko S. E. Russian Physics Journal, 2004, vol. 47, no. 5, pp. 556-562. Available at: https://link. springer.com/content/pdf/10.1023\%2FB\%3ARUPJ. 0000046330.92744.73.pdf

11. Savotchenko S. E. Proceedings of Voronezh State University. Series: Physics. Mathematics, 2016, no. 4, pp. 51-59. Available at: http://www.vestnik.vsu.ru/pdf/physmath/2016/04/2016-04-05.pdf (in Russ.)

12. Savotchenko S. E. Rus. Scientific Bulletins of BelSU. Ser: Mathematics. Physics, 2017, no. 20(269), pp. 79-85.
Available at: http://nv.bsu.edu.ru/nv/mag/06/archive/ (in Russ.)

13. Savotchenko S. E. Sov. JETP, 2018, vol. 153, no. 2, pp. 339-348. DOI: https://doi:10.7868/S0044451018020153

14. Savotchenko S. E. Proceedings of Voronezh State University. Series: Physics. Mathematics, 2018, no. 1, pp. 42-51. Available at: http://www.vestnik.vsu.ru/pdf/physmath/2018/01/2018-01-04.pdf (in Russ.)

15. Gerasimchuk I. V. Journal of Nano- and Electronic Physics, 2012. vol. 4, no. 4. pp. 04024-1-4. Available at: https://jnep.sumdu.edu.ua/ru/component/content/full_article $/ 883$

16. Gerasimchuk I. V., Gorbach P. K., Dovhopolyi P. P. Ukr. J. Phys., 2012, vol. 57, no. 6, pp. 678-683. Available at: http://archive.ujp.bitp.kiev.ua/files/journals/57/6/ 570614p.pdf.

17. Gerasimchuk I. V. Sov. JETP, 2015, vol. 121, no. 4, pp. 596-605. DOI: https://doi:10.7868/S0044451015100053

18. Savotchenko S. E. Modern Physics Letters B, 2018, vol. 32, no. 10 , pp. 1850120-12. DOI: https://doi. org/10.1142/S0217984918501208

19. Savotchenko S. E. Rus. Condensed Matter and Interphases, 2017, vol. 19, no. 4, pp. 567-572. Available at: http:// www.kcmf.vsu.ru/article.php?l=ru\&aid=855 (in Russ.)

20. Kosevich A. M. Sov. JETP, 1999, vol. 88, no. 1, pp. 168-173. Available at: https://link.springer.com/content/ pdf/10.1134\%2F1.558779.pdf.

21. Kosevich A. M. JETP Letters, 2001, vol. 73, no. 11, pp. 600-603. Available at: https://link.springer.com/content/ pdf/10.1134\%2F1.1392420.pdf

22. Savotchenko S. E. Russian Physics Journal, 2001, vol. 44, no. 4, pp. 412-419. DOI: https://doi:10.1023/ A:1011952514072

23. Savotchenko S. E. Russian Physics Journal, 2002, vol. 45 , no. 12 , pp. 1148-1158. DOI: https://doi:10.1023/ A: 1023858101297
Савотченко Сергей Евгеньевич - д. ф.-м. н, доцент, профессор кафедры высшей математики, Белгородский государственный технологический университет имени В. Г. Шухова; тел.: +7(920) 5610446, e-mail: savotchenkose@mail.ru
Sergey E. Savotchenko - Dr. Sci. (Phys.-Math.), Associate Professor, Professor of High Mathematics Department, Belgorod State Technological University named after V. G. Shukhov; tel.: +7(920) 5610446, e-mail: savotchenkose@mail.ru 